This item was submitted to Loughborough's Research Repository by the author.

Items in Figshare are protected by copyright, with all rights reserved, unless otherwise indicated.

\title{
Generation of undular bores in the shelves of slowly-varying solitary waves
}

PLEASE CITE THE PUBLISHED VERSION

LICENCE

CC BY-NC-ND 4.0

\section{REPOSITORY RECORD}

El, G.A., and Roger H.J. Grimshaw. 2019. "Generation of Undular Bores in the Shelves of Slowly-varying Solitary Waves". figshare. https://hdl.handle.net/2134/451. 


\title{
Generation of Undular Bores in the Shelves of Slowly-Varying Solitary Waves
}

\author{
G.A. $\mathrm{El}^{1}$ School of Mathematical and Information Sciences, Coventry University, \\ Priory Street, Coventry CV1 5FB, UK \\ R.H.J. Grimshaw Department of Mathematical Sciences, Loughborough University, \\ Loughborough LE11 3T, UK
}

\begin{abstract}
We study the long-time evolution of the trailing shelves that form behind solitary waves moving through an inhomogeneous media, within the framework of the variable-coefficient Korteweg-de Vries equation. We show that the nonlinear evolution of the shelf leads typically to the generation of an undular bore and an expansion fan, which form apart but start to overlap and nonlinearly interact after a certain time interval. The interaction zone expands with time and asymptotically as time goes to infinity occupies the whole perturbed region. Its oscillatory structure strongly depends on the sign of the inhomogeneity gradient of the variable background medium. We describe the nonlinear evolution of the shelves in terms of exact solutions to the KdV-Whitham equations with natural boundary conditions for the Riemann invariants. These analytic solutions, in particular, describe the generation of small 'secondary' solitary waves in the trailing shelves, a process observed earlier in various numerical simulations.
\end{abstract}

\section{Introduction}

The variable-coefficient Korteweg-de Vries (KdV) equation

$$
u_{t}+\alpha(t) u u_{x}+\lambda(t) u_{x x x}=0,
$$

where $\alpha(t), \lambda(t)$ are some given functions, is well-known as a model equation describing the propagation of weakly-nonlinear weakly-dispersive waves in inhomegeneous media. The physical problems described by (1) include shallow-water waves moving over an uneven bottom (Kakutani 1971, Johnson 1973), internal gravity waves in lakes of varying cross-section (Grimshaw 1978), long waves in rotating fluids contained in cylindrical tubes (Leibovich and Randall 1971), and many others. Typically, these problems imply that the coefficients in (1) are slowly varying in time so that $\sigma \sim\left|\lambda_{t}\right| \sim\left|\alpha_{t}\right| \ll 1$. Generally, the equation (1) is not integrable by the inverse scattering tranform method with some exeptions when the coefficients $\alpha(t)$ and $\gamma(t)$ satisfysome special relations (see Brugarino 1989, Dai 1999).

\footnotetext{
${ }^{1}$ On leave of absence from the Institute of Terrestrial Magnetism, Ionosphere and Radio Wave Propagation, Russian Academy of Sciences, Troitsk, Moscow Reg. 142190 Russia
} 
One of the most interesting effects modeled by (1) is the formation of a trailing shelf behind a slowly-varying solitary wave. Let the coefficients $\alpha(t)$ and $\lambda(t)$ be constant for $t<0$ and then change slowly starting from $t=0$. Suppose that a solitary wave solution of the constant-coefficient KdV equation (i.e. a soliton) is moving through the medium so that it reaches the point $x=0$ at $t=0$. It is clear that for $t>0$ it is no longer an exact solution of (1) and a slow time dependence should be introduced for its parameters (amplitude and phase)(see, for instance, Grimshaw 1979).

It is known (see Knickerbocker and Newell 1980, Newell 1985, Grimshaw and Mitsudera 1993, and the references therein) that although the slowly varying solitary wave provides conservation of momentum (to the first order in $\sigma$ )

$$
\frac{\partial}{\partial t}\left(\frac{1}{2} u^{2}\right)+\frac{\partial}{\partial x}\left(\frac{1}{3} \alpha(t) u^{3}+\lambda(t) u u_{x x}-\frac{1}{2} u_{x}^{2}\right)=0,
$$

it cannot provide conservation of mass (in the sense of the KdV equation)

$$
\frac{\partial}{\partial t} u+\frac{\partial}{\partial x}\left(\frac{1}{2} \alpha(t) u^{2}+\lambda(t) u_{x x}\right)=0 .
$$

To remedy this one has to to introduce a trailing shelf behind the solitary wave. The typical shelf amplitude is determined by the value of the inhomogeneity gradient $\sigma$ while its length should be of order $O\left(\sigma^{-1}\right)$, so that the shelf carries the mass $O(1)$. Depending on the sign of the inhomogeneity gradient, the shelf can have positive or negative polarity.

The formation and linear evolution of these shelves has been studied extensively (see the papers already cited and references therein). Up to times of order $O\left(\sigma^{-1}\right)$ the shelf has a stationary amplitude profile almost everywhere in the region between the point where the time variation of the coefficients has been switched on $(x=0)$ and the current position of the slowly varying solitary wave. Its length, therefore, changes with a speed of $O(1)$, and the transition to the unperturbed state at $x<0$ is achieved through a series of decaying oscillations described by the integral of Airy function (see, for instance, Knickerbocker and Newell, 1980). It is important that the shelf profile is completely determined by the the variation in the medium parameters.

In the case when the shelf has positive polarity it has been noted (see Newell 1985 ch. 3 ) that it can be decomposed (in the sense of the inverse scattering problem) into a large number of small solitons. These 'secondary' solitons should become visible at larger times $\left(t \gg \sigma^{-1}\right)$ when nonlinear effects in the shelf propagation would accumulate to compete with dispersive effects. Indeed, for a more rapid dependence of $\alpha(t)$ and $\lambda(t)$ on $t$, the generation of small solitons in the propagation of a solitary wave in an inhomogeneous medium can be observed more easily (see,for instance, Johnson 1972, 1997). Although this generation of secondary solitons from the elevation shelf has been long predicted, its analytic description has not been available until now. The reason for that is that the traditional approach to the problem when the shelf is treated as a perturbation in the inverse scattering transform formalism is seriously complicated by the necessity to deal with the large-scale potential (due to the extended length of the shelf). 
Such a potential, however, can be treated with the aid of the Semi-classical Lax-Levermore (1980) theory, which leads to the Whitham equations (Whitham 1965, 1974) for slowly varying wave parameters in the zero-dispersion limit. Later, Venakides (1985a) was able to extend the Lax-Levermore theory to the case of soliton-less initial data. The Whitham equations themselves have to be then integrated to reveal the dependence of the wave parameters on the initial data (the initial shape of the shelf in our case). The accuracy of this approach is naturally determined by the actual smallness of the medium inhomogeneity parameter $\sigma$.

The major assumption about the behaviour of the coefficients in (1) we make in this paper is the following: we suppose that the function $f(t)=\lambda(t) / \alpha(t)$ is a constant for $t<0$, then changes smoothly until some $t_{1}=O\left(\sigma^{-1}\right)$ and then is again a (different) constant. The asymptotic analysis made in Sec. 2, 3 of the present paper shows that in this case the process of formation of the shelf and that of its nonlinear evolution are substantially separated in time so that the nonlinear evolution of the shelf almost entirely happens in the region where $f(t)=$ constant. As we mentioned already, complete information about the function $f(t)$ is retained in the shelf shape, which serves as the large-scale initial data to the constantcoefficient KdV equation in this setting. The long-time KdV dynamics in this case is known to lead to the formation of an undular bore which starts with a soliton at its leading edge and then gradually degenerates into small-scale, linear oscillations at the opposite, trailing edge. The leading soliton of the undular bore can be identified with the 'secondary' shelf soliton observed in numerical simulations.

The first analytic solution to the Whitham equations describing evolution of the KdV undular bore was constructed by Gurevich and Pitaevskii (GP) (1974). The GP solution describes resolution of an initial step by a self-similar expanded undular bore. More general solutions have been constructed in the series of papers of Gurevich et. al. (1991, 1992, 1996) describing the evolution of monotonic and hump-like initial profile. The most general method for integrating the initial value problem for semi-classical KdV equation with arbitrary initial data has been developed recently by El, Krylov and Venakides (2001).

In this paper, we utilise some of the methods developed in the works just cited to describe analytically the formation and evolution of undular bores from the trailing shelves of slowlyvarying $\mathrm{KdV}$ solitary waves in terms of exact solutions to the Whitham equations.

\section{Asymptotic analysis}

First, it is convenient to introduce the new variables

$$
t^{\prime}=\int_{0}^{t} \alpha(\hat{t}) d \hat{t}, \quad \lambda^{\prime}(t)=\frac{\lambda}{\alpha} .
$$

Then, omitting the superscript "'", equation (1) becomes

$$
u_{t}+u u_{x}+\lambda(t) u_{x x x}=0 .
$$

Next, we assume that $\lambda$ is slowly varying, so that

$$
\lambda=\lambda(T), \quad T=\sigma t, \quad \sigma \ll 1 .
$$


Then the slowly-varying solitary wave asymptotic expansion is given by

$$
u=u_{0}+\sigma u_{1}+\ldots,
$$

where

$$
u_{0}=a \operatorname{sech}^{2}\left\{\gamma\left(x-\frac{\Phi(T)}{\sigma}\right)\right\}
$$

and

$$
\frac{d \Phi}{d T}=c=\frac{a}{3}=4 \lambda \gamma^{2}
$$

The variation of the amplitude $a$, the inverse-width parameter $\gamma$ and the speed $c$ with the slow time variable $T$ are determined by the momentum conservation law (2), so that,

$$
\int_{-\infty}^{\infty} \frac{1}{2} u_{0}^{2} d x=\text { constant }
$$

Substitution of (62) into(10) readily shows that

$$
\frac{\gamma}{\gamma_{0}}=\left(\frac{\lambda_{0}}{\lambda}\right)^{2 / 3}
$$

where the subscript ' 0 ' indicates quantities evaluated at $T=0$ say.

From (8), (9) and (11) it follows that the slowly-varying solitary wave, $u_{0}$ is now completely determined. However, as is now well-known (see, for instance, Grimshaw and Mitsudera 1993), $u_{0}$ by itself cannot conserve mass. Instead, the conservation of mass is assured by the generation of a trailing shelf $u_{s}$, which typically has an amplitude $O(\sigma)$ but stretches over a zone of $O\left(\sigma^{-1}\right)$, and hence has $O(1)$ mass. The law (3) for conservation of mass then shows that

$$
\int_{-\infty}^{\Phi / \sigma} u_{s} d x+\int_{-\infty}^{\infty} u_{0} d x=\text { constant }
$$

The second term on the left-hand side of (12) is readily found to be

$$
\frac{2 a}{\gamma}=24 \lambda \gamma=24 \lambda_{0} \gamma_{0}\left(\frac{\lambda}{\lambda_{0}}\right)^{1 / 3},
$$

on using (8), (9) and (11) in turn. Next, we assume that $u_{s}=\sigma q(X, T)$, where

$$
X=\sigma x, \quad T=\sigma t .
$$

Since $u_{s}$ must satisfy (5) it follows that

$$
q_{T}+\sigma q q_{X}+\sigma^{2} \lambda(T) q_{X X X}=0 .
$$

Next, on differentiating (12) with respect to $T$, we find that to leading order in $\sigma$,

$$
q=-\frac{2}{\gamma_{0}} \tilde{\lambda}_{T} \tilde{\lambda}^{-1 / 3} \equiv Q(T), \quad \text { on } X=\Phi(T)
$$


where $\tilde{\lambda}=\lambda / \lambda_{0}$. Now, 15), (16) together with (9) and (11) present a completely formulated boundary problem. Note that the small parameter $\sigma$ cannot be eliminated from the problem at this stage because the given function $\lambda(T)$ has a fixed time scale.

First we assume that $\lambda(T)$ is a smooth function and in particular has a continuous second derivative so that $Q(T)$ has a continuous first derivative. Then at least in some finite neighbourhood of the curve $X=\Phi(T)$ one can neglect the dispersive term in the $\mathrm{KdV}$ equation (15) and approximately describe the evolution by the Hopf equation

$$
q_{T}+\sigma q q_{X}=0
$$

with the same boundary condition,

$$
q=Q(T) \text { on } X=\Phi(T) .
$$

The solution to (17), (18) is readily found using the characteristics:

$$
q=Q\left(T_{0}\right), \quad X-\Phi\left(T_{0}\right)=\sigma Q\left(T_{0}\right)\left(T-T_{0}\right),
$$

where $T_{0}$ is a parameter along the initial curve $\Phi(T)$.

The expression (19) remains valid until neighbouring characteristics intersect and a breaking of the $q$-profile begins. This occurs when

$$
\Phi^{\prime}\left(T_{0}\right)+\sigma Q^{\prime}\left(T_{0}\right)\left(T-T_{0}\right)-\sigma Q\left(T_{0}\right)=0 .
$$

Since $\Phi^{\prime}=c>0$ and $\sigma \ll 1$, it follows that the singularity forms in finite time only if $Q^{\prime}\left(T_{0}\right)<0$ at least for some values of $T_{0}$. Let $T_{b}$ be the minimum value, as $T_{0}$ varies, such that $(20)$ is satisfied. Then the breaking singularity forms first at $T_{b}$ and the corresponding value $X_{b}$ is determined from (19). It can be easily seen from $(20)$ that $T_{b}=O(1 / \sigma)$ provided $Q^{\prime}\left(T_{0}\right)=O(1)$.

It is clear that in the vicinity of the breaking point $\left(X_{b}, T_{b}\right)$, the Hopf equation (17) no longer describes the evolution of $q$ adequately and the full equation (15) should be considered. As a result, the singularity is resolved by an undular bore, which is a 'dispersive' substitute for the common (dissipative) shock. The undular bore represents a modulated periodic wave train solution to (15), which provides resolution of a breaking singularity through generation of nonlinear small-scale oscillations. Since the work of Gurevich and Pitaevskii (1974) the structure of the undular bore is well established. It starts with the soliton at the leading edge and then, through the modulated 'cnoidal' wave regime, gradually degenerates into the vanishing amplitude linear wave at the opposite, trailing, edge.

As can be seen directly from the equation (15) the typical wavelength and the period of the nonlinear travelling wave should be

$$
\Delta X \approx \sigma^{1 / 2}, \quad \Delta T \approx 1 / \sigma^{1 / 2},
$$

while the typical spatio-temporal scales for the modulation parameters in the undular bore are:

$$
\Delta X_{\text {mod }}=O(1) \gg \Delta X, \quad \Delta T_{\text {mod }} \sim T_{b}=O\left(\frac{1}{\sigma}\right) \gg \Delta T .
$$


Here $\Delta T_{\text {mod }}$ serves also as a characteristic time of formation for the quasi-stationary structure of the undular bore (typical 'nonlinear' time in the system).

We note that both the typical period of nonlinear oscillations in the undular bore and the typical time of formation of the undular bore itself are much larger than the characteristic scale of change for the $\mathrm{KdV}$ coefficient $\lambda(T)$ (which is generally $O(1)$ ). This obviously happens due to the presence of the small coefficient $\sigma$ before the nonlinear term in (15). The above scale inconsistency would have made impossible the very existence of the quasistationary undular bore if $\lambda^{\prime}(T)$ was $O(1)$ for all $T$.

The usual assumption, however, is that $\lambda(T)$ changes smoothly within the finite interval $\left(0, T_{1}\right)$, say, from one constant value $\lambda_{0}$ to another one $\lambda_{1}$ i.e. the function $\lambda^{\prime}(T)$ has compact support. This physically reasonable assumption implies that the undular bore forms in the region where $\lambda(T)=\lambda_{1}$ because $T_{b} \gg T_{1}$. Therefore, once we are interested in the behaviour of slow modulations in the undular bore we essentially need to solve the constantcoefficient $\mathrm{KdV}$ equation with $\lambda(T)=\lambda_{1}$ while all the information about the variations of the function $\lambda(T)$ is completely retained in the boundary condition (16). At the same time, since $\Phi\left(T_{1}\right)-\Phi(0)=O(1)$ the boundary conditions (16) are spatially large-scale compared to the characteristic dimension of oscillations $\Delta X(21)$, which ensures applicability of the semi-classical Whitham approach in the sequel.

\section{Qualitative analysis of the wave patterns}

The preceding analysis assumes that $\tilde{\lambda}(T)$ is a smooth function, and in addition has a continuous second derivative, so that $Q(T)$ has a continuous first derivative (see (16)). However, in previous studies it has often been supposed that $\tilde{\lambda}(T)$ is a constant, 1 , for $T<0$ then varies smoothly for $0<T<T_{1}$ and is another constant $h$, for $T>T_{1}$. Thus, although $\tilde{\lambda}(T)$ can be assumed to be continuous for all $T$, there may exist discontinuities in $\tilde{\lambda}^{\prime}(T)$ at $T=0, T=T_{1}$ with consequent jump discontinuities in $Q(T)$. Strictly speaking the preceding asymptotic analysis should not be used in the vicinity of $T=0$ and $T=T_{1}$. Nevetheless, it is instructive to assume that the slowly varying solitary wave analysis holds for $0<T<T_{1}$, and to explore the consequences of the predicted jump discontinuities in $Q$ at $T=0$ and $T=T_{1}$. Note that from (16), $Q$ is zero for $T<0$ and $T>T_{1}$.

The qualitative picture of the evolution strongly depends on whether $h<1$ or $h>1$. The principal difference between these two cases becomes obvious if we consider the shelf as a potential in the quantum mechanical Schrödinger equation associated with (15) when $\lambda=$ constant. The case $h<1$ corresponds to the elevation shelf $(Q>0)$ a nd implies a big number $\left(N \sim \sigma^{-1 / 2}\right)$ of solitons as an asymptotic result of the evolution of the shelf as $T \rightarrow \infty$. The solitons correspond to the contribution of discrete spectrum in the associated scattering problem. The negative shelf $(h>1, Q<0)$, on the contrary, eventually evolves into a modulated linear wave packet (continuous spectrum in the associated scattering problem). Despite this drastic contrast as $T \rightarrow \infty$, at intermediate times $\left(T \sim \sigma^{-1}\right)$, both elevation and depression shelves represent certain combinations of two waves, one of which is an undular bore while another one is an expansion fan. As a result, both cases can be described in 
mathematically equivalent terms using the semi-classical Whitham approach.

Let us consider the wave pattern in the 'multisoliton' case, $h<0$, in more detail. To be specific, we take the function $\tilde{\lambda}(T)$ in a physically reasonable form (Fig. 1a),

$$
\begin{aligned}
& 1 \quad T<0 \\
& \begin{array}{cl}
\tilde{\lambda}(T)=(1-p T)^{3 / 2} & 0 \leq T \leq T_{1} \\
h & T>T_{1},
\end{array}
\end{aligned}
$$

where $h=\left(1-p T_{1}\right)^{3 / 2}, p>0, T_{1}<1 / p$. This implies very simple behaviour for $Q(T)$ :

$$
\begin{aligned}
& T<0 \\
& Q(T)=3 p / \gamma_{0} \quad 0 \leq T \leq T_{1} \\
& 0 \quad T>T_{1},
\end{aligned}
$$

The boundary curve $X=\Phi(T)$ in the region $\left(0, T_{1}\right)$ is readily found from (9), (11)(we put $\Phi(0)=0$ ),

$$
\Phi(T)=\frac{8 \lambda_{0} \gamma_{0}^{2}}{p}\left(1-(1-p T)^{1 / 2}\right) .
$$

However, since we are interested in the nonlinear evolution of the shelf (time scale $O\left(\sigma^{-1}\right)$ ) we can project the curve (25) onto the $X$-axis, and keeping the same accuracy replace the boundary conditions (16) with the initial data in the form of a rectangular pulse (Fig. 1b)

$$
q(X, 0)=q_{0}(X)=\begin{array}{ll}
0 & X<0 \\
3 p / \gamma_{0} & 0 \leq X \leq l \\
0 & X>l,
\end{array}
$$

where $l=\Phi\left(T_{1}\right)=\frac{8 \lambda_{0} \gamma_{0}^{2}}{p}\left(1-\left(1-p T_{1}\right)^{1 / 2}\right)$. Without any loss of generality one can put $p=\gamma_{0} / 3$ so that $q_{0}=1$ in the interval $(0, l)$ and $q_{0}=0$ outside this interval. The solution for arbitrary $p$ can be then obtained by simple rescaling

$$
q \rightarrow \frac{3 p}{\gamma_{0}} q, \quad t \rightarrow \frac{3 p}{\gamma_{0}} t
$$

Thus, we have two jump discontinuities at $X=0$ and $X=l$. The discontinuity at $X=0$ evolves into a self-similar expansion fan (region I in Fig.1c) while the discontinuity at $X=l$ yields the self-similarly expanding undular bore (region III in Fig. 1c). There is a constant plateau (region II in fig. 1c) between the expansion fan and the undular bore. The width of the plateau decreases with time and vanishes at $T=T^{*}$ when the leading edge of the expansion fan intersects the trailing front of the undular bore and the motion ceases to be self-semilar due to nonlinear-wave interaction. Further, the interaction zone expands with time and asymptotically occupies the entire perturbed region. Eventually, as $T \rightarrow \infty$ it transforms into a chain of weakly interacting solitons ordered by their amplitude. In Sec.5 we shall construct the asymptotically exact solution to the initial value problem (15), (26) describing the above process. 
The case $h>1$ (and, therefore, $p<0$ in $(23)-(26)$ ) can be qualitatively analysed in a similar way (see Fig.3). As was mentioned above, the final result of the evolution in this case would be a modulated vanishing-amplitude linear wave packet to correlate with the predictions of the associated semi-classical scattering theory for the potential barrier (see Venakides 1985a, El and Khodorovskii 1993). The corresponding asymptotically exact solution will be constructed in Sec. 6 .

It should be noted that our choice of the special behaviour for $\lambda(T)(23)$ is not conditioned by any constraints of the methods we are going to use below but rather is motivated by the greater simplicity of presentation.

\section{The Whitham equations and boundary conditions}

We are going to study the small dispersion $\mathrm{KdV}$ equation in the form

$$
q_{T}+\sigma q q_{X}+\sigma^{2} \lambda_{1} q_{X X X}=0 .
$$

with the initial data in the form of a rectangular pulse

$$
q_{0}(X)= \pm 1 \text { if } 0<X<l \text {, and }=0 \text { for } X<0, X>l
$$

The dimensionless dispersion parameter of the problem is

$$
\epsilon=\left(\sigma \lambda_{1}\right)^{1 / 2} / l \ll 1
$$

The rigorous analysis of problems of this type based on the semi-classical asymptotics in the inverse scattering problem solution (Lax and Levermore 1983, Venakides 1985a,b) shows that the asymptotic solution in a certain region of the $(X, T)$ plane is described by the one-phase modulated travelling solution of (28), while the modulations are governed by the Whitham equations (Whitham 1965). This is exactly the region of the undular bore described earlier by Gurevich and Pitaevskii (1974) in the same terms, but with more heuristic reasoning.

The travelling wave solution of the KdV equation (28) is parametrised by three constants of integration $r_{1} \leq r_{2} \leq r_{3}$ and is expressed in terms of Jacobi elliptic function $\mathrm{cn}(\Theta ; m)$

$$
q(X, T)=a \operatorname{cn}^{2}\left[\left(\frac{a}{12 m}\right)^{1 / 2} \frac{X-U \sigma T}{\left(\sigma \lambda_{1}\right)^{1 / 2}} ; m\right]+d
$$

where

$$
\begin{gathered}
a=2\left(r_{2}-r_{1}\right), \quad m=\frac{r_{2}-r_{1}}{r_{3}-r_{1}}, \quad d=r_{3}-r_{2}+r_{1}, \\
U=\frac{1}{3}\left(r_{1}+r_{2}+r_{3}\right) .
\end{gathered}
$$

Here $a$ is the amplitude of the travelling wave, $m$ is the modulus and $\sigma U$ is the phase velocity. The wavelength of the travelling wave (31) is expressed in terms of the complete 
elliptic integral of the first kind $K(m)$ :

$$
L=\left(6 \sigma \lambda_{1}\right)^{1 / 2} \int_{r_{1}}^{r_{2}} \frac{d \xi}{\sqrt{\left(\xi-r_{1}\right)\left(\xi-r_{2}\right)\left(\xi-r_{3}\right)}}=\left(6 \sigma \lambda_{1}\right)^{1 / 2} \frac{2 K(m)}{\sqrt{r_{3}-r_{1}}}
$$

Then if we allow the constants of integration $r_{j}$ to be functions of $X, \tau=\sigma T$ they become the Riemann invariants of the Whitham system describing slow modulations of the travelling wave (31) (Whitham 1965),

$$
\partial_{\tau} r_{j}+V_{j}(\mathbf{r}) \partial_{X} r_{j}=0, \quad j=1,2,3
$$

where the characteristic velocities $V_{j}(\mathbf{r})$ are computed as certain combination of complete elliptic integrals (see Gurevich and Pitaevskii 1974). Here we will use more compact representation for $V_{j}$ emphasizing their meaning as 'nonlinear group velocities' found independently by Gurevich, Krylov and El (1991), and Kudashev (1991),

$$
V_{j}=\frac{\partial_{j} \omega}{\partial_{j} k}=U-\frac{1}{3} \frac{L}{\partial_{j} L} .
$$

Here $\partial_{j} \equiv \partial / \partial r_{j}, k(\mathbf{r})=2 \pi / L$ is the wavenumber, and $\omega(\mathbf{r})=k U$ is the frequency. The representation (36) follows from the wave number conservation law

$$
k_{\tau}+\omega_{x}=0
$$

considered as a consequence of the diagonal system (35) (see also El, Grimshaw and Pavlov 2001)

Outside the undular bore where the flow is smooth the solution to the KdV equation (15) is asymptotically equivalent to the solution of the Hopf equation $q \approx r(X, \tau)$

$$
r_{\tau}+r r_{X}=0
$$

with the same initial condition (29).

The Whitham equations are then subject to the special boundary conditions which provide $C^{1}$-smooth matching of the 'inner' Riemann invariants $r_{j}$ with the 'outer' Riemann invariant $r$. It is clear that since the number of the 'inner' equations (35) is equal to three while outside we have the only Hopf equation (38), the boundaries where the matching happens should be the multiple (double) characteristics of the Whitham system for the solution to be found. This coupling, as can be easily seen from (36), can happen only when either $r_{2} \rightarrow r_{3}$ or $r_{2} \rightarrow r_{1}$. The first case corresponds to the soliton limit $(m \rightarrow 1)$ in the travelling wave solution (31) while the second one yields the vanishing-amplitude sinusoidal wave $m \rightarrow 0$. Therefore, the desired solution to the modulation equations describing the undular bore should provide the gradual transition of the nonlinear travelling wave (31) from the soliton at the leading edge $X^{+}$, say, to the linear wave at the opposite, trailing, edge $X^{-}$. Both edges, naturally, are unknown at the onset and should be determined along with the solution. It can be deduced from (36) that in both soliton and linear limits, the third, uncoupled, characteristic velocity transforms into that of the Hopf equation (38) which provides 
the natural matching with the smooth 'outer' solution on boundaries $X^{ \pm}(\tau)$. The desired natural boundary conditions thus take the form (Gurevich and Pitaevskii 1974),

$$
\begin{array}{ll}
r_{2}\left(X^{+}, \tau\right)=r_{3}\left(X^{+}, \tau\right), & r_{1}\left(X^{+}, \tau\right)=r\left(X^{+}, \tau\right), \\
r_{2}\left(X^{-}, \tau\right)=r_{1}\left(X^{-}, \tau\right), & r_{3}\left(X^{-}, \tau\right)=r\left(X^{-}, \tau\right) .
\end{array}
$$

\section{$5 \quad$ Evolution of the elevation shelf}

As was shown in Sec. 3 the, the asymptotic description as $\epsilon \rightarrow 0$ of the nonlinear evolution of the positive shelf is reduced to the initial value problem for the small-dispersion $\mathrm{KdV}$ equation (28). At each moment, the domain of determination of the solution on the $(X \tau)$ plane is split into three regions where the solution has different forms. Outside these domains $q=0$. There are two cases to consider

a) $\tau<\tau *=l / 2$. This time interval corresponds to the initial stage of nonlinear evolution of the positive rectangular profile (29) (see Fig. 2a, Fig. 3). We consider the three aforementioned spatial regions as follows.

(I) $\quad 0<X<X_{f}$.

This region is occupied by the expansion fan emanating from the point $X=0, \tau=0$. The approximate $\mathrm{KdV}$ solution in this region coincides with the self-similar solution of the Hopf equation (38)

$$
q \approx r(X, \tau)=\frac{X}{\tau}
$$

The weak discontinuities at the edges of the expansion fan $X=0$ and $X=X^{f}=\tau$ can be resolved by linear oscillatory wave trains(see Gurevich and Pitaevskii 1974)

(II) $\quad X^{f}<X<X^{-}$

The plateau region with constant flow $q=1$ stretches between the leading edge of the expansion fan $X^{f}$ and the trailing edge of the undular bore $X^{-}$

(III) $\quad X^{-}<X<X^{+}$

The region is occupied by the undular bore emanating from the point $X=l$. The modulations in the undular bore are described by the Whitham system (35) subject to the boundary conditions (39), (40), which in our case take the form (see Fig.2a)

$$
r_{2}\left(X^{+}\right)=r_{3}\left(X^{+}\right), \quad r_{1}\left(X^{+}\right)=0, \quad r_{2}\left(X^{-}\right)=r_{1}\left(X^{-}\right), r_{3}\left(X^{-}\right)=1 .
$$

The corresponding solution has been found by Gurevich and Pitaevskii (1974) and represents the simple wave solution to the Whitham system:

$$
r_{1}=0, \quad r_{3}=1 \quad X=V_{2}\left(0, r_{2}, 1\right) \tau+l
$$

It can be easily obtained from (36) that

$$
V_{2}\left(0, r_{2}, 1\right)=\frac{1+r_{2}}{3}-\frac{2}{3} \frac{r_{2}\left(1-r_{2}\right)}{\mu\left(r_{2}\right)-\left(1-r_{2}\right)},
$$


where $\mu(m)=E(m) / K(m), E(m)$ is the complete elliptic integral of the second kind. Then we have for the edges of the undular bore

$$
X^{+}=V_{2}(0,1,1) \tau+l=\frac{2}{3} \tau+l, \quad X^{-}=V_{2}(0,0,1) \tau+l=l-\tau .
$$

We note that $X^{+}(\tau)$ is the trajectory of the leading soliton in the undular bore, which has the amplitude $a=2$.

It can be seen that at the point $\left(X^{*}, \tau^{*}\right)=(l / 2, l / 2)$ the leading edge of the expansion fan intersects the trailing edge of the undular bore and after this an interaction zone arises the solution is no longer described by the self-similar formulas (41) or (43).

b) $\tau>\tau^{*}=l / 2$

The solution in the domains I and III, as before, is described by formulas (41) and (43) respectively, but instead of the plateau II we have now the zone of a nonlinear interaction of the undular bore and the expansion fan (see Fig. 2b, Fig. 3).

(II) $X^{-}<X<X^{f}$

The solution in the interaction zone is the same travelling wave (31) but the Whitham modulations (35) are now subject to somewhat different boundary conditions. At the trailing edge $X^{-}$of the interaction zone (which is the edge where oscillations vanish as well as in the case of the 'pure' undular bore the solution of the Whitham equations should match the expansion fan solution (41), so that

$$
r_{2}\left(X^{-}\right)=r_{1}\left(X^{-}\right)=0, \quad r_{3}\left(X^{-}\right)=r\left(X^{-}\right),
$$

while at the opposite, leading, edge $X^{f}(\tau)$ we have (see Fig. 2b)

$$
r_{2}\left(X^{f}-0\right)=r_{2}\left(X^{f}+0\right), r_{3}\left(X^{f}\right)=1, \quad r_{1}\left(X^{f}\right)=0 .
$$

Here we have omitted the $\tau$-dependence of $r_{j}$ 's for brevity. It is clear that the problem (35),(45),(46) requires two Riemann invariants $r_{2}, r_{3}$ to vary while $r_{1}=0$ remains the constant part of the solution. The $2 \times 2$ system

$$
\partial_{\tau} r_{2}+V_{2}\left(0, r_{2}, r_{3}\right) \partial_{X} r_{2}=0, \quad \partial_{\tau} r_{3}+V_{3}\left(0, r_{2}, r_{3}\right) \partial_{X} r_{3}=0
$$

can be linearized by a standard hodograph transformation in which dependent and independent variables change their places. The hodograph equations for $X\left(r_{2}, r_{3}\right)$ and $\tau\left(r_{2}, r_{3}\right)$, provided $\partial_{X} r_{j} \neq 0$, are

$$
\partial_{2} X-V_{2}\left(0, r_{2}, r_{3}\right) \partial_{2} \tau=0, \quad \partial_{3} X-V_{3}\left(0, r_{2}, r_{3}\right) \partial_{3} \tau=0 .
$$

Here as earlier $\partial_{j} \equiv \partial_{r_{j}}$.

We make in (48) the change of variables

$$
x-V_{j} \tau=W_{j} \quad j=2,3 .
$$

which leads to a symmetric system for $W_{j}\left(r_{2}, r_{3}\right)$ :

$$
\frac{\partial_{i} W_{j}}{W_{i}-W_{j}}=\frac{\partial_{i} V_{j}}{V_{i}-V_{j}} ; \quad i \neq j ; i, j=2,3 .
$$


The construction (49), (50) is known as the generalized hodograph transform and was originally designed for integration of so-called semi-Hamiltonian hydrodynamic type systems with the number of dependent variables exceeding that of independent ones (Tsarev 1985). However, Tsarev's method turns out to be useful even in the case of $(2 \times 2)$ systems when it is formally equivalent to the classic hodograph transform.

Now we have to transform the natural boundary conditions (45), (46) for the Riemann invariants $r_{j}(X, \tau)$ into the boundary conditions for the functions $W_{j}\left(r_{2}, r_{3}\right)$ on the hodograph plane. For that, we consider the relationship (49) on the trailing edge of the interaction zone. For $j=3$, taking into account the first condition (45), it takes the form

$$
x-r_{3} \tau=W_{3}\left(0, r_{3}\right) .
$$

According to the second condition (45) the invariant $r_{3}$ should match the solution of the Hopf equation (41) on the trailing edge . Comparing (51) with (41) we get

$$
W_{3}\left(0, r_{3}\right)=0 \text {. }
$$

The second boundary condition for the leading edge of the interaction zone can be obtained for the function $W_{2}$ by matching of (49) for $j=2$ with the GP solution (43) which readily gives

$$
W_{2}\left(r_{2}, 1\right)=l \text {. }
$$

One can see that the conditions (52), (53) are those of Goursat type for the hyperbolic system (50) (see, for instance, Tricomi 1961) because the lines $r_{j}=$ constant are the characteristics of $(50)$.

Thus, instead of the nonlinear system (47) with the nonlinear boundary conditions (39), (40) (the boundaries $X^{ \pm}$themselves depend on the solution) we have arrived at the equivalent linear system (50) with the linear boundary conditions (51), (53) on known boundaries. Equations (50) despite their linearity still have too complicated coefficients to be integrated directly. Although they allow separation of variables, which reduces the problem to solving some ordinary differential equations, the solution can be obtain in this only in the form of tan infinite series (Gurevich, Krylov and Mazur 1989). Here we will make use of the method proposed by Gurevich, Krylov, and El (1991, 1992) to obtain the solution in a closed form.

We notice that the functions $W_{j}$ and $V_{j}$ enter the system (50) in a completely symmetric way. Then one can suggest a substitution for $W_{j}$ inspired by the representation (36) for the characteristic velocities $V_{j}$

$$
W_{j}=\frac{\partial_{j}(k f)}{\partial_{j} k}=f-\frac{L}{\partial_{j} L} \partial_{j} f,
$$

where $f\left(r_{2}, r_{3}\right)$ is a new unknown function, which serves as a potential for $W_{j}$. Substituting (54), (36), (34) into (50) provided $r_{1}=0$ readily yields the Euler-Darboux-Poisson (EDP) equation (see for instance Tricomi 1961) for the potential $f$,

$$
2\left(r_{3}-r_{2}\right) \partial_{23}^{2} f=\partial_{2} f-\partial_{3} f
$$

The equation EDP has also been obtained in a different way by Kudashev and Sharapov (1991) and Tian (1993). 
Substituting (54) into the boundary conditions (52), (53) we obtain a set of ordinary differential equations for the boundary values of the function $f\left(r_{1}, r_{2}\right)$ :

$$
f\left(r_{2}, 1\right)-\frac{K\left(r_{2}\right)}{K^{\prime}\left(r_{2}\right)} \partial_{2} f\left(r_{2}, 1\right)=l, \quad f\left(0, r_{3}\right)+2 r_{3} \partial_{3} f\left(0, r_{3}\right)=0
$$

Equations (56) are readily integrated to give

$$
f\left(r_{2}, 1\right)=c_{1} K\left(r_{2}\right)+l, \quad f\left(0, r_{3}\right)=\frac{c_{2}}{\sqrt{r_{3}}} .
$$

Here $c_{1,2}$ are arbitrary constants, which appear in the boundary conditions due to the introduction of the potential representation (54) and their choice is not restricted by any conditions. It is convenient to put $c_{1}=0$.

Now we represent the general solution to the EDP equation (55) in the form (see, for instance, Tricomi 1961)

$$
f=\int_{0}^{r_{2}} \frac{\phi_{1}(\beta) d \beta}{\sqrt{\beta\left(r_{3}-\beta\right)\left(\beta-r_{2}\right)}}+\int_{r_{3}}^{1} \frac{\phi_{2}(\beta) d \beta}{\sqrt{\beta\left(\beta-r_{3}\right)\left(\beta-r_{2}\right)}},
$$

where $\phi_{1,2}(\beta)$ are arbitrary (generally, complex-valued) functions. Substitution of (58)into the first boundary condition (57) gives the Abel integral equation for $\phi_{1}(\beta)$ (see, for instance, Courant and Hilbert 1962)

$$
\int_{0}^{r_{2}} \frac{\phi_{1}(\beta) d \beta}{\sqrt{\beta(1-\beta)\left(\beta-r_{2}\right)}}=l .
$$

The inverse Abel transform yields

$$
\phi_{1}(\beta)=\frac{l}{\pi} \sqrt{1-\beta}
$$

Then substituting (58), (60) into the second boundary condition (57) we get

$$
\frac{l}{\sqrt{r_{3}}}+\int_{r_{3}}^{1} \frac{\phi_{2}(\beta) d \beta}{\beta \sqrt{\left(\beta-r_{3}\right)}}=\frac{c_{2}}{\sqrt{r_{3}}}
$$

Choosing $c_{2}=l$ we get $\phi_{2} \equiv 0$ and the desired solution becomes

$$
\begin{aligned}
& f\left(r_{2}, r_{3}\right)=\frac{l}{\pi} \int_{0}^{r_{2}} \sqrt{\frac{1-\beta}{\beta\left(r_{2}-\beta\right)\left(r_{3}-\beta\right)}} d \beta \\
& =\frac{2 l}{\pi \sqrt{\left(1-r_{2}\right) r_{3}}} \Pi_{1}\left(\frac{r_{2}}{1-r_{2}} ; \frac{r_{2}\left(1-r_{2}\right)}{r_{3}\left(1-r_{2}\right)}\right),
\end{aligned}
$$

where $\Pi_{1}(\varphi ; s)$ is the complete elliptic integral of the third kind. The dependence of the $r_{j}$ 's on $X$ and $\tau$ is then reconstructed with the aid of (49), (54). 
Now given the solution (62) one can find the curves $X^{-}(\tau), X^{f}(\tau)$, which are the boundaries of the interaction zone in physical $(X, \tau)$ plane. The trailing edge $X^{-}(\tau)$ according to (45), (36) represents the double characteristics of the Whitham system (47) for the obtained solution as $r_{2}=0$, i.e.

$$
\frac{d X^{-}}{d \tau}=V_{1}\left(0,0, r_{3}\right)=V_{2}\left(0,0, r_{3}\right)=-r_{3},
$$

Then taking into account that on the trailing edge $r_{3}$ matches with the solution to the Hopf equation (63) we get $X^{-}=C / \tau$. Since the point of onset of the interaction zone is $X=l / 2, \tau=l / 2$ we obtain

$$
X^{-}=\frac{l^{2}}{4 \tau} \quad\left(\tau \geq \frac{l}{2}\right) .
$$

The leading edge $X^{f}(\tau)$ of the interaction zone is determined from $(49,(54)$, (62) at $r_{3}=1$. The result is represented in a parametric form

$$
\begin{aligned}
X^{f} & =\frac{l}{2 \sqrt{1-m}}\left(1-\frac{3 m(1-m)}{\mu(m)(1+m)-(1-m)}\right)+l, \\
\tau^{f} & =\frac{3 l}{2 \sqrt{1-m}}\left(1-\frac{m \mu(m)}{\mu(m)(1+m)-(1-m)}\right) .
\end{aligned}
$$

Now we consider the asymptotics of the obtained solution as $\tau \gg 1$. It follows from (49) that

$$
\tau=\frac{W_{3}-W_{2}}{V_{3}-V_{2}}
$$

Straightforward analysis of (66)for the obtained solution shows that $\tau \rightarrow \infty$ implies $m \rightarrow 1$ for all $X$,

$$
\tau \approx \frac{3 l}{4 \pi \sqrt{r_{3}\left(1-r_{3}\right)}} \ln (1-m) \gg 1 .
$$

This means that asymptotically as $\tau \rightarrow \infty$ the oscillations in the interaction zone transform into weakly interacting solitons (the result predictable from the point of view of the inverse scattering problem).

The asymptotics for the leading edge of the interaction zone is obtained from (65) as $m \rightarrow 1$

$$
X^{f}=\frac{2}{3} \tau+l-\frac{9 l^{2}}{16 \tau} \ln \tau+O\left(\frac{1}{\tau}\right),
$$

i.e. $X^{f}$ becomes asymptotically close to the leading edge of the undular bore $X^{+}(44)$ while the trailing edge $(64) X^{-} \rightarrow 0$. Therefore, zone II asymptotically occupies the whole perturbed region (see Fig.3).

The asymptotics of the solution (49) as $\tau \rightarrow \infty$ y yield as well the amplitude distribution for the solitons

$$
a=2 r_{3}=3 s+\frac{2 X_{0}(s)}{\tau}+O\left(\frac{1}{\tau^{2}}\right), \quad s=\frac{X}{\tau} .
$$


Here

$$
X_{0}(s)=\frac{l}{\pi \tau}\left(\sqrt{\frac{2-3 s}{3 s}} \ln \left(1-\frac{3 s}{2}\right)+\pi-2 \operatorname{atan} \sqrt{\frac{2-3 s}{3 s}}\right) .
$$

We note that according to (68) $0<s<2 / 3$. The first term of the decomposition (69) describes a 'triangle' distribution for the velocities of noninteracting particles (solitons) (see Whitham 1974, ch. 17) while the second one represents the correction connected with the weak interaction between solitons due to finite (although large) distance between them.

For the wavelength (the distance distribution between the weakly interacting solitons) we get

$$
L_{s} \approx \frac{8 \pi \tau}{l} \sqrt{\frac{\sigma \lambda_{1}}{6}(1-a)} .
$$

We note that (70) can be obtained directly from the Bohr-Sommerfeld formula for the semiclassical distribution of the discrete spectrum of the potential $q_{0}(X)$ (Karpman 1967, see also, Whitham 1974, ch. 17).

\section{Evolution of the depression shelf}

Technically, the study of the evolution of the depression shelf $\left(q_{0}(X)=-1\right.$ for $\left.0<X<l\right)$ is completely analogous to that made in the previous section for the elevation shelf. Again, we have two cases to consider .

a) $0<\tau<\tau^{*}=3 l / 2$.

There are three regions (see Fig. 5a, Fig. 6) where the solution has different forms.

(I) $X^{-}<X<X^{+}$

This region is occupied by the undular bore emanating from the point $(0,0)$. It is described by the GP self-similar solution analogous to (43) but with different values of the constant invariants (see Fig.5a)

$$
r_{1}=-1, \quad r_{3}=0 \quad X=V_{2}\left(-1, r_{2}, 0\right) \tau .
$$

The trailing and the leading edges of the undular bore are correspondingly

$$
X^{-}=-2 \tau, \quad X^{+}=-\frac{1}{3} \tau .
$$

(II) $X^{+}<X<X^{f}$

The plateau region $q=-1$ (see Fig.5a).

(III) $X^{f}<X<l$

The expansion fan emanating from the point $(0 . l)$

$$
q \approx r(X, \tau)=-\frac{l-X}{\tau} .
$$

Its trailing edge coordinate is $X^{f}=l-\tau$. One can see that at the point $(-l / 2,3 l / 2)$ the leading edge of the undular bore meets the trailing edge of the expansion fan and a nonlinear 
interaction zone analogous to that described in previous section forms. The qualitative behaviour of modulations in the interaction zone as $t \rightarrow \infty$, however, appears to be completely different.

b) $\tau>3 l / 2$

Again, the solutions in the domains (I) and (III) remain as (71), (73). The modulations in the interaction zone $X^{f}<X<X^{+}$are described by the Whitham system (35) with the boundary conditions (see Fig.5b)

$$
\begin{gathered}
r_{2}\left(X^{+}\right)=r_{3}\left(X^{+}\right)=0, \quad r_{1}\left(X^{+}\right)=r\left(X^{+}\right), \\
r_{2}\left(X^{f}-0\right)=r_{2}\left(X^{f}+0\right), \quad r_{1}\left(X^{f}\right)=-1, \quad r_{3}\left(X^{f}\right)=0
\end{gathered}
$$

It is obvious that $r_{3}=0$ is the solution. Then we apply to the remaining two Whitham equations $j=1,2$ the same chain of substitutions described in the previous section and as a result arrive at the EDP equation for $f\left(r_{1}, r_{2}\right)$ with the boundary conditions of the Goursat type (cf. (57)):

$$
f\left(-1, r_{2}\right)=0 \quad f\left(r_{1}, 0\right)=\frac{c}{\sqrt{-r_{1}}}+l,
$$

where $c$ is an arbitrary constant. It is convenient now to represent the general solution to the EDP equation in the form

$$
f=\int_{r_{1}}^{r_{2}} \frac{\phi_{1}(\beta) d \beta}{\sqrt{\beta\left(r_{2}-\beta\right)\left(\beta-r_{1}\right)}}+\int_{r_{2}}^{0} \frac{\phi_{2}(\beta) d \beta}{\sqrt{\beta\left(\beta-r_{2}\right)\left(\beta-r_{1}\right)}}
$$

where the functions $\phi_{1,2}(\beta)$ are found by the substitution of (77) into the boundary conditions (76). As a result we get

$$
\begin{aligned}
& f\left(r_{1}, r_{2}\right)=l-\frac{l}{\pi} \int_{r_{2}}^{0} \sqrt{\frac{1+\beta}{-\beta\left(\beta-r_{2}\right)\left(\beta-r_{1}\right)}} d \beta \\
& =l-\frac{2 l}{\pi \sqrt{-r_{1}\left(1+r_{2}\right)}} \Pi\left(-\frac{r_{2}}{1+r_{2}} ; \frac{r_{2}\left(1+r_{2}\right)}{r_{1}\left(1+r_{2}\right)}\right) .
\end{aligned}
$$

Although this solution for the potential $f$ looks very similar to that for the elevation shelf case (62) it leads to a completely different asymptotics for the sought functions $r_{1,2}(X, \tau)$ after their reconstruction with the aid of (54), (49). Before we proceed with asymptotics, we obtain the edges of the interaction zone.

The leading front $X^{+}$according to (74), (36)represents the double characteristics of the Whitham system for the obtained solution as $r_{2}=0$, i.e

$$
\frac{d X^{+}}{d \tau}=V_{2}\left(r_{1}, 0,0\right)=V_{3}\left(r_{1}, 0,0\right)=\frac{r_{1}}{3}
$$


Then taking into account that on the leading edge $r_{1}$ matches with the solution to the Hopf equation (73) we get $X^{+}=C \tau^{1 / 3}+l$. Since the interaction zone originates now at the point $X=-l / 2, \tau=3 l / 2$ we obtain

$$
X^{+}=l-\left(\frac{3 l}{2}\right)^{2 / 3} \tau^{1 / 3}, \quad \tau \geq \frac{3 l}{2} .
$$

The leading edge as earlier represents the trajectory of the leading soliton in the undular bore. However, in contrast to the case of the elevation shelf this soliton asymptotically dies down with time due to the interaction with the expansion fan. Its amplitude

$$
a_{s}=-2 r_{1}=2 \frac{l-X^{+}}{\tau}=\left(\frac{3 l}{2 \tau}\right)^{4 / 3}, \quad \tau \geq \frac{3 l}{2} .
$$

The trailing edge $X^{f}(\tau)$ of the interaction zone is determined from (49), (54), (78) at $r_{1}=-1$. The result is represented in a parametric form

$$
\begin{aligned}
X^{f} & =-\frac{l}{2 \sqrt{m}}\left(1+\frac{3(1-m) m}{\mu(m)(2-m)-2(1-m)}\right), \\
\tau^{f} & =\frac{3 l}{2 \sqrt{m}}\left(1+\frac{(1-m)(1-\mu(m))}{\mu(m)(2-m)-2(1-m)}\right) .
\end{aligned}
$$

Straightforward analysis of the solution $X-V_{1,2} \tau=W_{1,2}$ shows that as $\tau \rightarrow \infty$ we have $m \rightarrow 0$

$$
\tau \approx \frac{32 l \sqrt{-r_{1}\left(1+r_{1}\right)}}{\pi r_{1}^{2}} \frac{1}{a^{2}} \gg 1
$$

i.e. the oscillations in the interaction zone asymptotically convert into the linear wave train $(a \rightarrow 0)$, which again is in exact accordance with the predictions of the inverse scattering transform problem for the purely reflective potentials. Still, despite the asymptotically as $\epsilon \rightarrow 0$ zero contribution of the discrete spectrum, solitons do appear in the solution for the depression shelf as a part of an intermediate asymptotics and then gradually fade away. For $r_{1}(X, \tau)$ we get the asymptotic formula

$$
X=2 r_{1} \tau+2 \sqrt{\frac{2 l}{\pi}}\left(-r_{1}\left(r_{1}+1\right)\right)^{1 / 4} \tau^{1 / 2}+O(\ln \tau) .
$$

The asymptotics of the trailing edge $X^{f}$ of the interaction zone is obtained from (82) as $m \rightarrow 0$ :

$$
X^{f}=-2 \tau+\frac{7 l^{2 / 3}}{2^{4 / 3}} \tau^{1 / 3}+O\left(\frac{1}{\tau^{1 / 3}}\right),
$$

The amplitude distribution in the linear wave train is readily obtained from (83), (84)

$$
a(X, \tau)=2\left(r_{2}-r_{1}\right) \approx \frac{4}{\tau^{1 / 2}} \sqrt{\frac{2 l}{\pi}}(-s(1+s))^{1 / 4}, \quad s=\frac{X}{2 \tau}<0
$$


The formula (86) is in accordance with the momentum conservation law for the linear waves, which requires $a \sim \tau^{-1 / 2}$. For the wavenumber $k=2 \pi / L$ (see (34)) we get the decomposition

$$
\sigma \lambda_{1} k^{2}=\frac{X}{3 \tau}-\frac{1}{6} a(X, \tau)+O(1 / \tau) .
$$

The leading term of this decomposition describes motion of the linear wave packet with the group velocity $\omega^{\prime}(k)=3 \sigma \lambda_{1} k^{2}$ according to the linear dispersion relation for the $\mathrm{KdV}$ equation (28).

\section{Conclusion}

In this article, we have considered the problem of nonlinear evolution of the trailing shelves forming behind solitary waves in weakly inhomogeneous media. The problem is described by the variable-coefficient KdV equation. Due to the special scaling in the problem the process of formation of the shelf and that of its nonlinear dispersive evolution appear to be substantially separated in time. If the variation of the KdV coefficients occurs within a finite time interval, the boundary-value problem for the shelf evolution for large ('nonlinear') times is reduced to the initial value problem for the constant-coefficient small dispersion KdV equation while the temporal dependence of the coefficients is absorbed into the initial data.

We have shown that the nonlinear evolution of the shelf is accompanied by formation of an undular bore and an expansion fan, which start to overlap and nonlinearly interact after a certain time interval. The leading soliton of the undular bore is identified with the 'secondary' solitary wave predicted by earlier numerical simulations. The asymptotic result of the evolution depends on the sign of the inhomogeneity gradient. The elevation shelf eventually transforms into a large number of small solitons while the depression shelf converts into a linear wave train.

We have described the whole process in terms of the exact analytic solutions to the KdVWhitham equations describing behaviour of slow modulations in the nonlinear travelling waves. The solutions are obtained with the aid of the generalised hodograph transform in a 'potential' form, which reduces the original nonlinear problem of Gurevich-Pitaevskii type (albeit not self-similar) to the classic Goursat problem for the Euler-Darboux-Poisson equation. The solutions of the Goursat problem are then reconstructed back into the $X, \tau$ dependence of the wave parameters.

The authors would like to thank V. Khodorovskii for useful discussions. The work of G.A.E was partially supported by the Russian Foundation for Basic Research (RFBR) under the grant \# 00-01-00210.

\section{References}

[1] Brugarino, T. 1989 Painlevé property, auto-Bäcklund transformation, Lax pairs, and reduction to the standard form for the Korteweg-de Vries equation with nonuniformities. J.Math. Phys. $301013-1015$. 
[2] Courant, R. and Hilbert, D. 1962 Methods of mathematical physics. Vol.II: Partial differential equations. Wiley, New York.

[3] Dai, H.-H. 1999 Exact solutions of a variable-coefficient KdV equation arising in a shallow water J.Phys. Soc. Japan 68 1854-1858.

[4] El, G.A. and Khodorovskii, V.V. 1993 Evolution of a solitonless perturbation in Korteweg - de Vries hydrodynamics Phys. Lett. A 182 49-52.

[5] El, G.A., Grimshaw, R.J.H. and Pavlov, M.V. 2001 Integrable shallow water equations and undular bores. Stud. Appl. Math. 106 157-186. 75-86.

[6] El, G.A., Krylov, A.V., Venakides, S. 2001 Unified approach to KdV modulations. Comm. Pure Appl. Math. 54 1243-1270.

[7] Grimshaw, R.H.J. 1978 Long nonlinear waves in channels of arbitrary cross-section J.Fluid Mech. 86 415-451.

[8] Grimshaw, R. 1979 Slowly varying solitary waves. I Korteweg - de Vries equation Proc.Roy.Soc. A 368 359-375.

[9] Grimshaw, R. and Mitsudera, H. 1993 Slowly varying solitary wave solutions of the perturbed Korteweg - de Vries equation revisited. Stud. Appl. Math. 90 75-86.

[10] Gurevich, A.V. and Pitaevsky, L.P. 1974 Nonstationary structure of a collisionless shock wave. Sov.Phys. JETP 38 291-297.

[11] Gurevich, A.V., Krylov, A.L. and Mazur, N.G. 1989 Quasisimple waves in Korteweg de Vries hydrodynamics Sov. Phys. JETP 68 966-974.

[12] Gurevich, A.V., Krylov, A.L., and El, G.A. 1991 Riemann wave breaking in dispersive hydrodynamics. JETP Lett. 54 102-107.

[13] Gurevich, A.V., Krylov, A.L. and El, G.A. 1992 Evolution of a Riemann wave in dispersive hydrodynamics. Sov. Phys. JETP 74 957-962.

[14] Gurevich, A.V., Krylov, A.L., Khodorovskii, V.V. and El, G.A. 1996 Supersonic flow past finite-length bodies in dispersive hydrodynamics JETP 82 709-718.

[15] Johnson, R. 1972 Some numerical solutions of a variable-coefficient Korteweg - de Vries equation (with application to solitary wave development on a shelf) J.Fluid Mech. $\mathbf{5 4}$ 81-91.

[16] Johnson, R.S. 1973 On an asymptotic solution of the Korteweg - de Vries equation with slowly varying coefficients. J.Fluid Mech. 60 813-824.

[17] Johnson, R.S. 1997 A modern introduction to the mathematical theory of water waves. CUP, Cambridge, 445pp. 
[18] Kakutani, T. 1971 Effect of uneven bottom on gravity waves, J.Phys. Soc. Japan 30 $272-275$.

[19] Kaup, D.J. and Newell, A.C. 1978 Solitons as particles, oscillators, and in slowly changing media. Proc. Roy. Soc. A $361412-446$.

[20] Karpman, V.I. 1967 An asymptotic solution of the Korteweg - de Vries equation. Phys. Lett. A 25 708-709.

[21] Knickerbocker, C.J. and Newell, A.C. 1980 Shelves and the Korteweg - de Vries equation. J.Fluid Mech. 98 803-818.

[22] Kudashev, V.R. 1991 "Waves-number conservation" and succession of symmetries during a Whitham averaging, JETP Lett. 54 175-178.

[23] Kudashev, V.R. and Sharapov, S.E. 1991 Inheritance of KdV symmetries under Whitham averaging and hydrodynamic symmetries of the Whitham equations. Theor. Math. Phys. 87 358-363.

[24] Lax, P.D. and Levermore, C.D. 1983 The small dispersion limit of the Korteweg - de Vries equation I, II, III, Comm. Pure Appl. Math. 36 253-290, 571-593, 809-829.

[25] Leibovich, S. and Randall, J.D. 1971 Solitary waves in concentrated vortices, J.Fluid Mech. $51625-635$.

[26] Newell, A.C., 1985 Solitons in mathematics and physics. SIAM, Philadelphia, 244pp.

[27] Tian, F.R. 1993 Oscillations of the zero dispersion limit of the Korteweg - de Vries equation. Comm. Pure Appl. Math. 46 1093-1129.

[28] Tricomi, F. 1961 Differential equations. Blackie and Sons, Boston.

[29] Tsarev, S.P. 1985 On Poisson brackets and one-dimensional systems of hydrodynamic type Soviet Math. Dokl. 31 488-491.

[30] Venakides, S. 1985a The zero dispersion limit of the Korteweg - de Vries equation for initial potentials with nontrivial reflection coefficient. Comm. Pure Appl. Math. 38 $125-155$.

[31] Venakides, S. 1985b The generation of modulated wave trains in the solution of the Korteweg - de Vries equation, Comm. Pure Appl. Math. 38 883-909.

[32] Whitham, G.B. 1965 Non-linear dispersive waves, Proc. Roy. Soc. A283 238-291.

[33] Whitham, G.B. 1974 Linear and Nonlinear Waves, Wiley, New York, 636pp. 


\section{Figure Captions}

- 1. Elevation shelf

a)variable $\mathrm{KdV}$ coefficient $\lambda(T)$, b)initial data for the shelf evolution problem,

c) distribution $q(X)$ for $0<\tau<\tau^{*}$

- 2. Riemann invariant behaviour for the elevation shelf

a) $0<\tau<\tau^{*}$, b) $\tau>\tau^{*}$

- 3. Splitting of the $(X, \tau)$ plane for the elevation shelf

- 4. Depression shelf

a)variable $\mathrm{KdV}$ coefficient $\lambda(T)$, b)initial data for the shelf evolution problem,

c) distribution $q(X)$ for $0<\tau<\tau^{*}$

- 5. Riemann invariant behaviour for the depression shelf

a) $0<\tau<\tau^{*}$, b) $\tau>\tau^{*}$

- 6. Splitting of the $(X, \tau)$ plane for the depression shelf 
Fig 1

a)

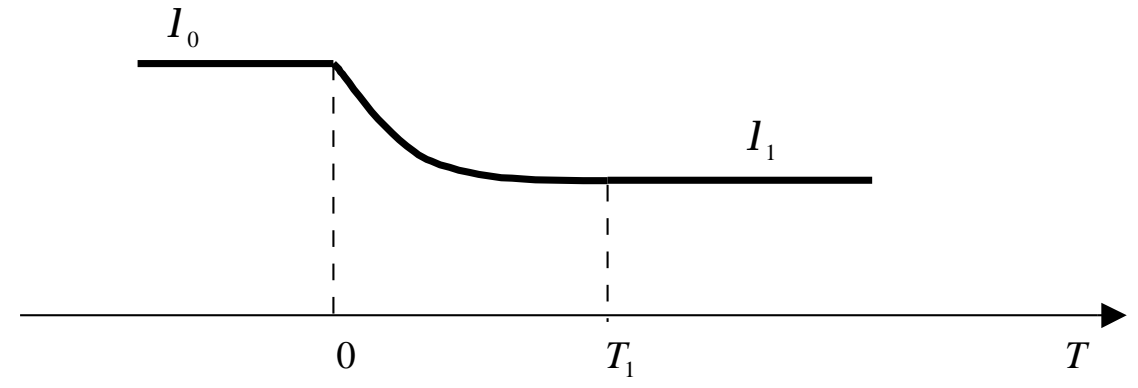

b)

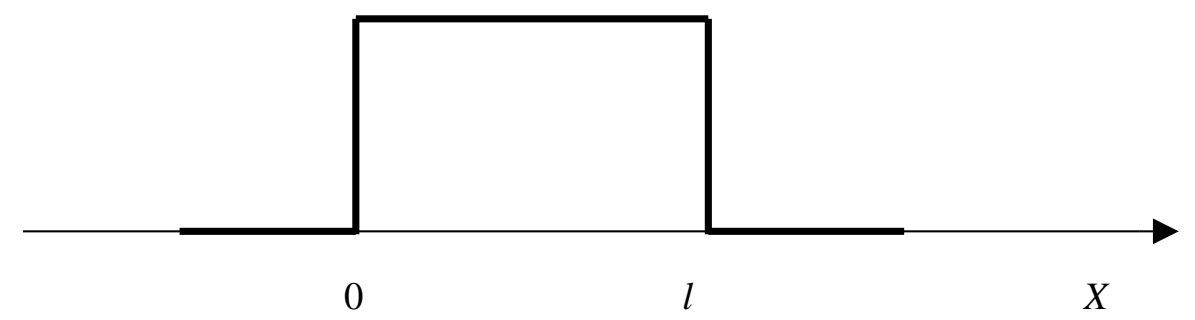

c)

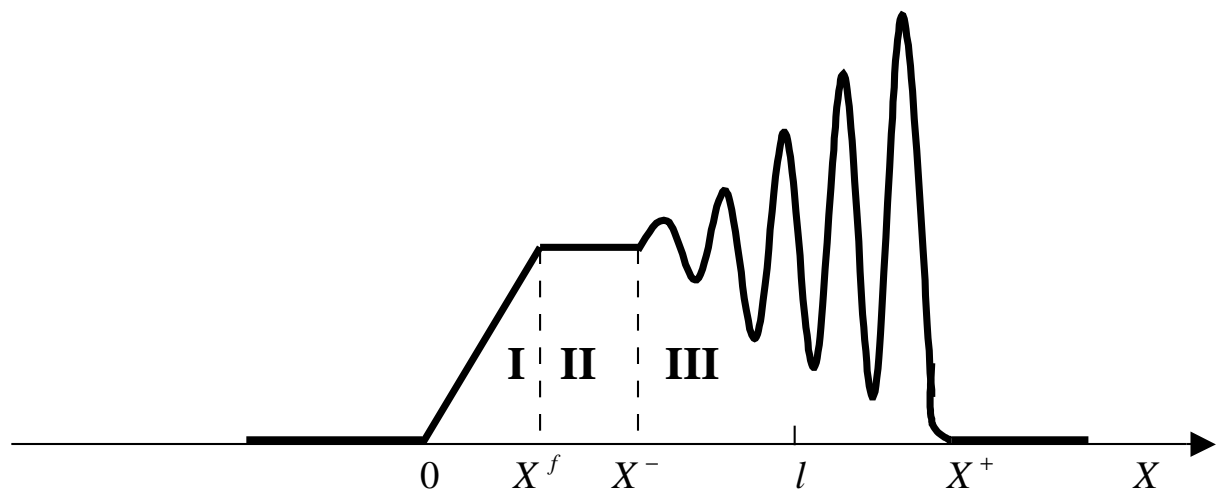


Fig.2

a)

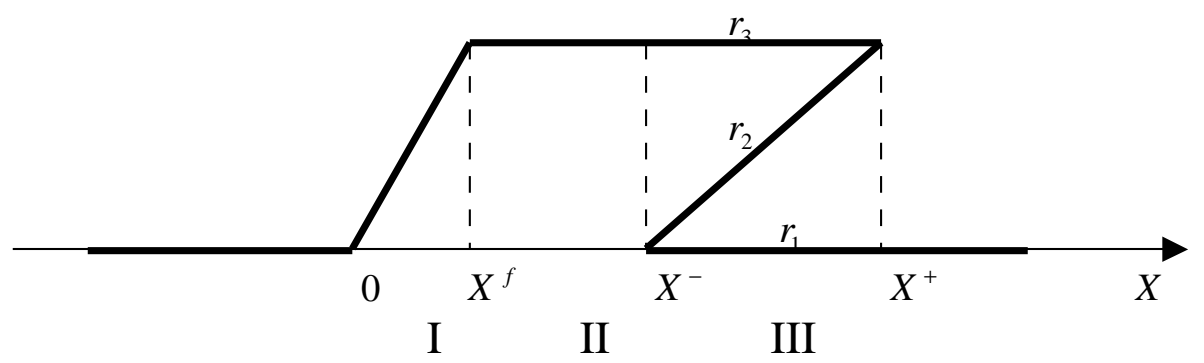

b)

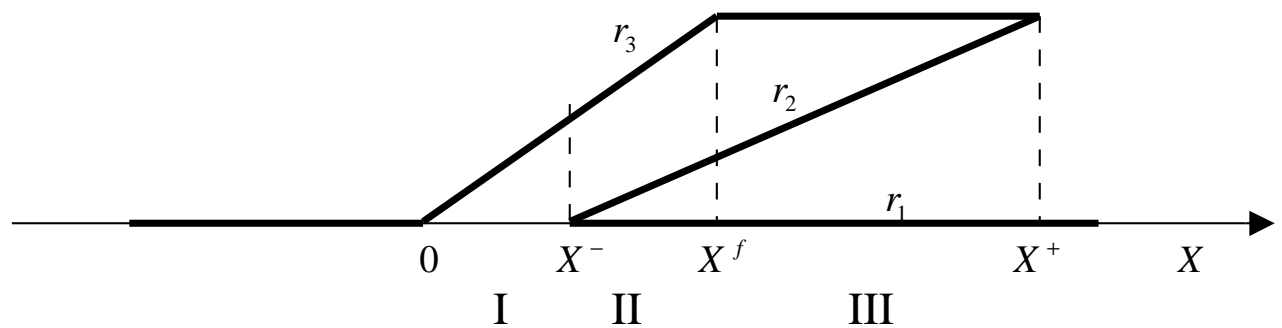


Fig.3

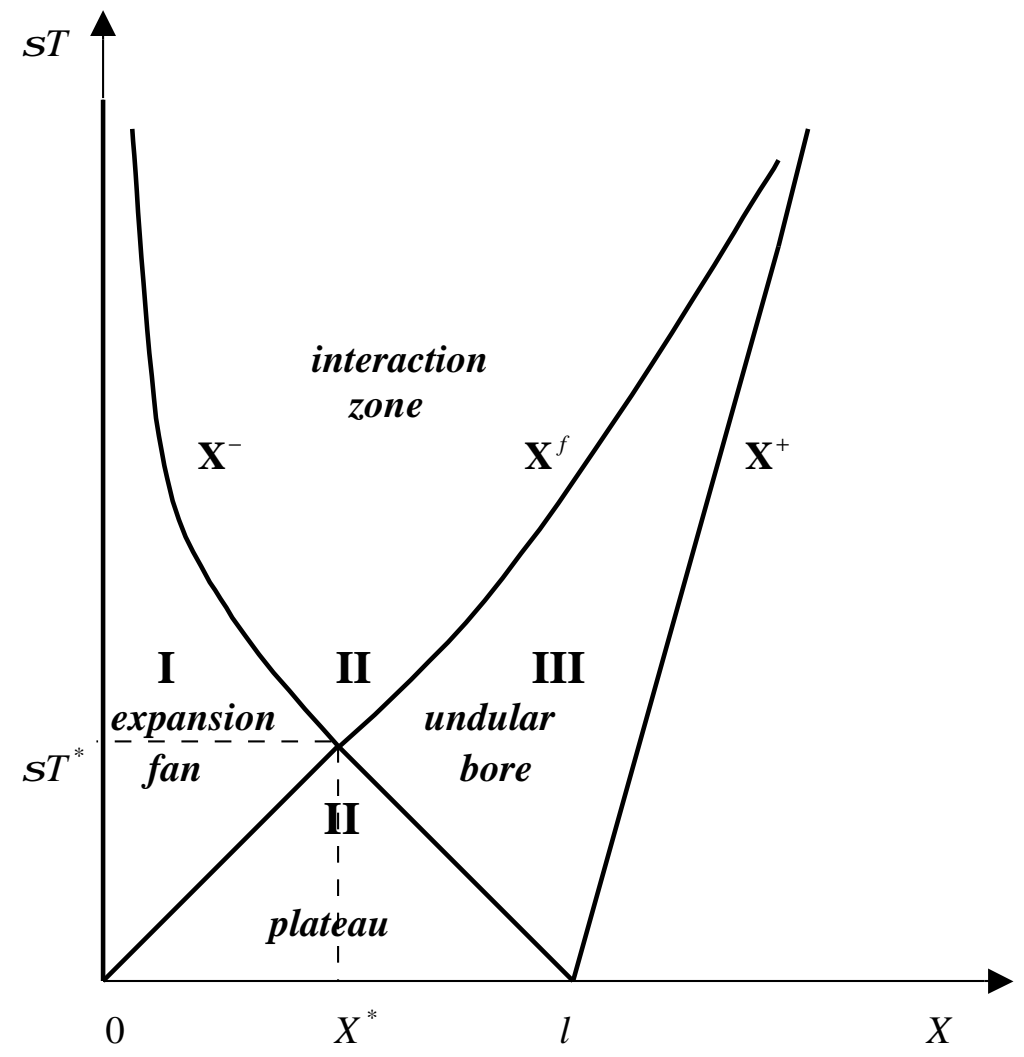


Fig 4

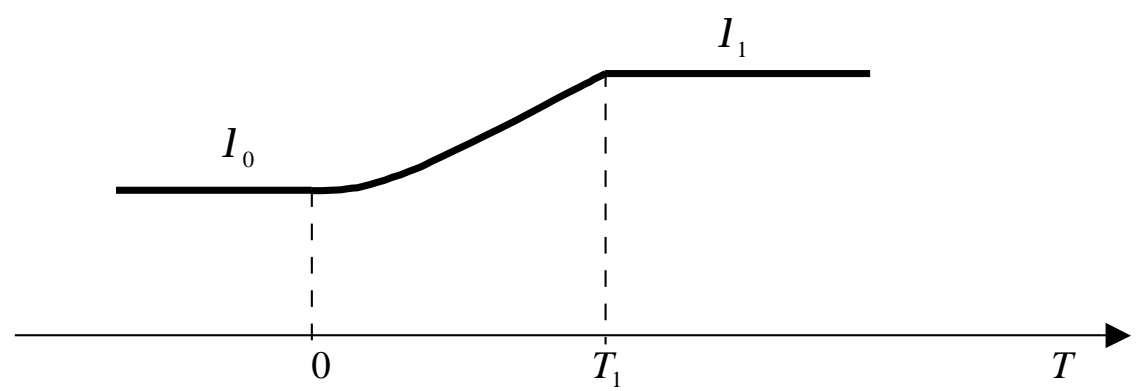

b)

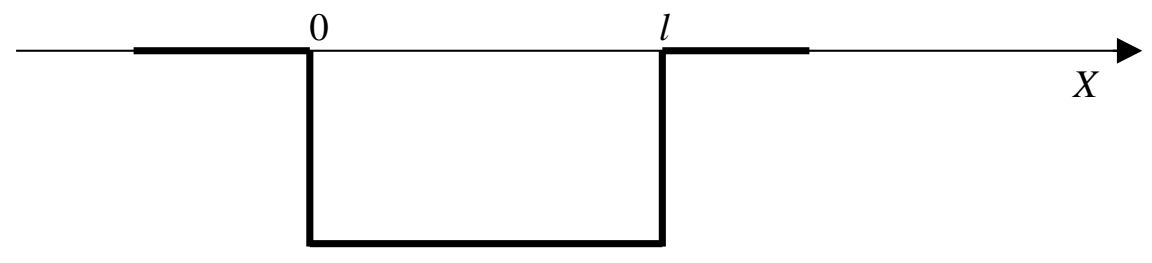

c)

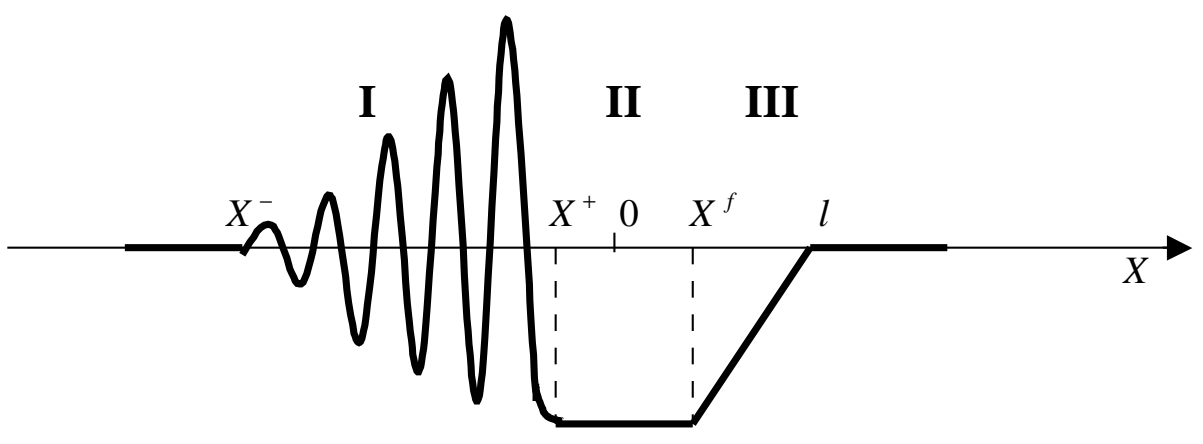


Fig.5

a)

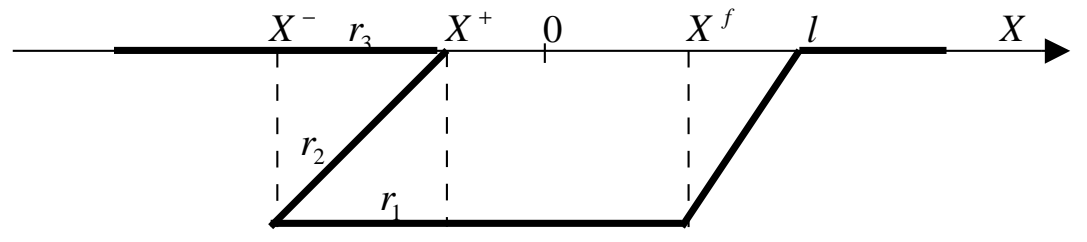

b)

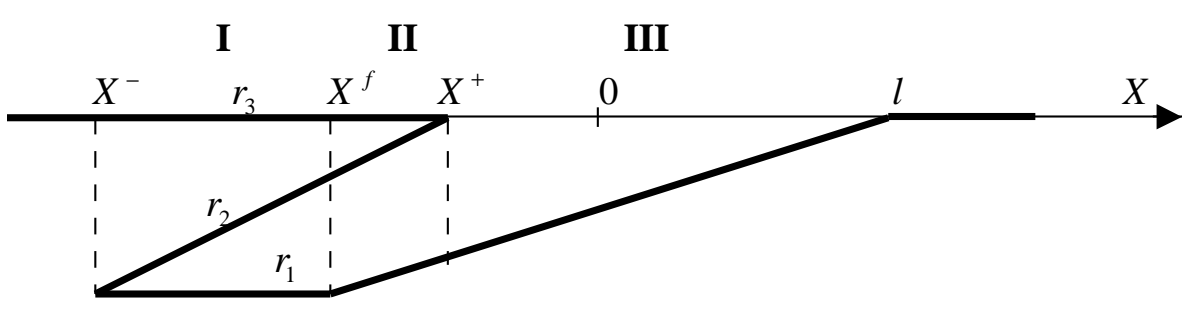


Fig.6

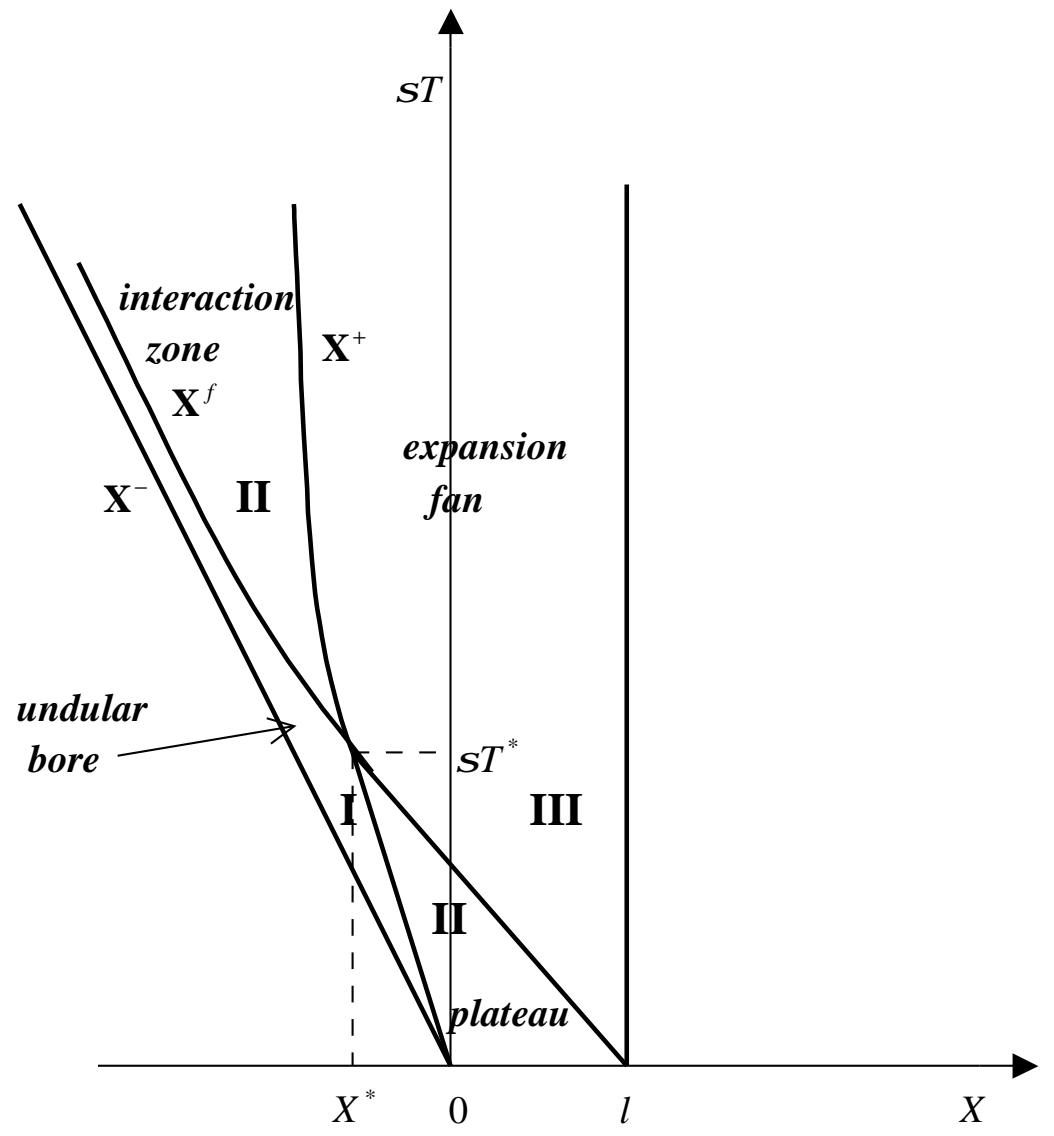

